\title{
Caracterización de aspectos relacionados con la utilización de los medicamentos fiscalizados en droguerias y farmacias-droguerias de Medellín y el área metropolitana
}

\author{
Characterization of aspects related to the use of controlled \\ drugs in Drugstores and pharmacies-drugstores \\ in Medellin and the metropolitan area
}

Mauricio Ceballos ${ }^{1}$; José-Alejandro Giraldo ${ }^{1}$; Víctor-Hugo Marín²; Pedro Amariles ${ }^{1}$

Forma de citar: Ceballos M, Giraldo J, Giraldo J, Marín V, Amariles P. Caracterización de aspectos relacionados con la utilización de los medicamentos fiscalizados en droguerías y farmacias-droguerías de Medellín y el área metropolitana. Rev Univ Ind Santander Salud. 2018; 50(1): 27-36. doi: http://dx.doi.org/10.18273/revsal.v50n1-2018003 (c) (1)

\section{Resumen}

Introducción: Los fallos prevenibles en la cadena del medicamento se relacionan con los Problemas Relacionados con la Utilización de Medicamentos, y están asociados a errores de medicación (prescripción, dispensación, administración o uso), incluyendo los fallos en el Sistema de Suministro de Medicamentos (disponibilidad y calidad). Objetivo: Identificar información básica de los Establecimientos Farmacéuticos (EF), caracterizar algunos procesos relacionados con la utilización de medicamentos fiscalizados (MF), incluyendo cumplimiento de la normatividad, y determinar el nivel de percepción del riesgo y las necesidades de educación continua del personal farmacéutico (PF). Metodología: Se realizó un estudio de corte transversal. Se utilizó un cuestionario sobre los procesos enfocados en la utilización de MF, durante mayo a septiembre de 2016. Resultados: Se visitaron 700 establecimientos y se encuestaron a igual cantidad de PF. El 88\% (617) de los establecimientos eran de estratos socioeconómicos bajo y medio. Los MF más dispensados fueron: amitriptilina 91,4\% (640), tramadol 90\% (630) y trazodona 60\% (419). El 71,6\% (501) de los encuestados consideró que el expendedor de drogas es el personal más idóneo para dispensar MF. El 25\% (177) de los encuestados percibieron como muy deficiente a regular el proceso de información y educación que ellos brindan, y el 32\% (223) con igual percepción en la responsabilidad que tienen con el uso y resultados en salud de los pacientes. Conclusiones: Se identifica la información general de los establecimientos, al igual que de los procesos relacionados con la utilización de MF. La percepción de los encuestados sobre el personal idóneo para la dispensación correcta de MF podría ser inadecuado. Un alto porcentaje del PF percibe falencias de educación enfocada en la utilización correcta de los MF.

Palabras clave: Farmacia; Farmacéuticos; Utilización de medicamentos; Dispensación de productos; Medicamentos controlados.

1. Universidad de Antioquia, Medellín, Colombia.

2. Laboratorios Laproff S.A. Medellín, Colombia.

Correspondencia: Javier Mauricio Ceballos. Dirección: Calle 67 \#53-108, Bloque 2 Oficina 125. Correo electrónico: javier.ceballos@udea.edu. co. Teléfono: +4 2195460 . 


\begin{abstract}
Introduction: Preventable failures in the drug chain are related to Drugs Problems Related Use and are associated with medication errors (prescription, dispensing, administration or use by the patient or caregiver), including failures In the Medication Supply System (availability and quality). Objective: Recognize basic information of the Pharmaceutical Establishments (PE), to characterize some processes related to the use of controlled drugs (CD), including compliance with the regulations, and determine the level of risk perception and the continuing education needs of the staff pharmacy. Methodology: A cross-sectional, epidemiological study was performed. A questionnaire was used on the processes focused on the use of CD, between May to September of 2016. Results: $700 \mathrm{PE}$ were visited and an equal number of staff pharmacy were interviewed. Eighty-eight percent (617) of PE are socioeconomic low and medium status, and have an average of 15.3 years of operation (SD 13.6 years). The most favored CD were: mitriptyline 91.4\% (640), tramadol 90\% (630) and trazodone 60\% (419). 76.6\% (536) of the respondents considered that the Pharmacy Technician is the most suitable personnel to dispense CD. 25\% (177) of the respondents perceived as very poor to regulate the process of information and education that they provide, and 32\% (223) with equal perception in the responsibility that they have with the use and results in patients' health. Conclusions: It identifies the general information of $\mathrm{PE}$, as well as of processes related to the use of $\mathrm{CD}$. The perception of respondents about the SP that is suitable for the correct dispensing of CD may be inadequate. A high percentage of the SP perceives educational failures focused on the correct use of CD.
\end{abstract}

Keywords: Pharmacy; Pharmacists; Products commerce; Drug utilization; Controlled drugs.

\section{Introducción}

En Colombia, la propuesta de una Política Farmacéutica Nacional (PFN) en el $2003^{1}$, actualizada y complementada en el $2012^{2}$, propone el desarrollo y la prestación de Servicios Farmacéuticos (SF) ajustado al modelo de servicios de salud, dentro del sistema y en el ámbito comercial, y cuyo propósito es optimizar la utilización de los medicamentos, reducir las inequidades en el acceso y asegurar la calidad de los mismos en el marco del Sistema de Seguridad Social en Salud.

El Decreto 2200 en el año 2005, incorporado en el Decreto 780/2016, se expidió soportado en la PFN del 2003, y establece que los SF deben cumplir cuatro objetivos; a) buscar, promover y propiciar estilos de vida saludables, b) prevenir factores de riesgo derivados del uso inadecuado de medicamentos y dispositivos médicos, c) promover su uso adecuado, y d) ofrecer Atención Farmacéutica a los pacientes, trabajando en equipo con los demás profesionales de la salud, además, define a las Droguerías y las Farmacias-Droguerías como Establecimientos Farmacéuticos (EF) minoristas, que realizan, entre otros, procesos de recepción, almacenamiento y dispensación de medicamentos y dispositivos médicos, y la elaboración de preparados magistrales a nivel ambulatorio (exclusivo para Farmacias-Droguerías) $^{3}$. El número de $\mathrm{EF}$ ambulatorios ha ido creciendo paulatinamente. Según datos del estudio de caracterización ocupacional del sector farmacéutico en Colombia $^{4}$, para el 2003 existían en el país 14.208 Droguerías y alrededor de 1.000 Farmacias-Droguerías, de estas, 1.209 se encontraban en Antioquia.
La cadena del medicamento se refiere al conjunto de los sucesivos eslabones desde su registro o aprobación para su comercialización hasta su empleo por el usuario final. Cada uno de estos eslabones es un determinante de los efectos finales del medicamento sobre la salud del paciente en particular y de la comunidad en general, y por tanto, el acto médico realizado por el prescriptor no es el único determinante en los resultados de salud ${ }^{5}$, por ello, el personal que labora en los SF debe contribuir con la implementación de estrategias farmacéuticas que busquen detectar, prevenir y resolver las desviaciones en los objetivos terapéuticos esperados con el uso de los medicamentos, inclusive, este aporte se deberá efectuar en varios puntos de la cadena del medicamento. Estas estrategias deben asegurar el control de factores que pongan en riesgo la vida del paciente con los medicamentos que este vaya a utilizar o que ya está utilizando (máxima efectividad y seguridad) ${ }^{6,7}$.

En Colombia, los fallos prevenibles en la cadena del medicamento se asocian con los Problemas Relacionados con la Utilización de Medicamentos (PRUM), que corresponden a causas prevenibles de problemas relacionados con medicamentos, asociados a errores de medicación (prescripción, dispensación, administración o uso por parte del paciente o cuidador), incluyendo los fallos en el Sistema de Suministro de Medicamentos (disponibilidad), principalmente por la ausencia en los servicios de procesos administrativos y técnicos que garanticen la existencia de medicamentos que realmente se necesiten, acompañados de las características de efectividad, seguridad, calidad de la información y educación necesaria para su utilización 
correcta $^{3}$. Dentro del Sistema de Suministro, la selección de los medicamentos se ha considerado como uno de los procesos más relevantes con relación al acceso y disponibilidad, y está relacionado con el análisis de la eficacia, efectividad, seguridad y evaluación económica de los medicamentos, garantizando que los medicamentos escogidos son los que realmente se requieren para atender los problemas de salud más prevalentes en la población, además, se ajusta a la realidad de las cantidades necesarias de acuerdo a la oferta y la demanda ${ }^{8}$.

Complementario a esto, El National Coordinating Council for Medication Error Reporting and Prevention (NCC MERP) define los errores de medicación como: cualquier evento prevenible que pueda causar daño al paciente o dé lugar a una utilización inapropiada de los medicamentos, cuando estos están bajo el control de los profesionales de la salud, del paciente o del consumidor. Estos eventos pueden estar relacionados con la práctica profesional, con los productos, los procedimientos y los sistemas de salud, incluyendo fallos en la prescripción, comunicación, etiquetado, envasado, denominación, preparación, dispensación, distribución, administración, educación, seguimiento y uso ${ }^{9}$. Por tanto, las causas asociadas a la aparición de eventos adversos se observan a través de todo el proceso de utilización de los medicamentos, iniciando desde la selección, la adquisición, el almacenamiento, la prescripción, la dispensación, y el uso, como se describe, y se extiende hasta el seguimiento (monitorización) de la efectividad y seguridad del medicamento en el paciente.

Los medicamentos se utilizan para prevenir, diagnosticar y curar enfermedades; sin embargo, su uso puede causar problemas de salud, situación que se considera como uno de los mayores problemas de salud, entre otras causas, por la aparición de efectos no deseados para el paciente y a la no consecución de los objetivos terapéuticos, además, al aumento de los costos de la atención en salud ${ }^{10,11}$. Un amplio grupo de estos, son los Medicamentos Fiscalizados (MF), que contienen sustancias reguladas por convenios internacionales y se utilizan, entre otras, en el tratamiento del dolor, urgencias obstétricas, trastornos mentales y neurológicos ${ }^{12}$.

En Colombia, la Resolución 1478 de $2006^{13}$, contiene el listado de sustancias sometidas a fiscalización y los productos farmacéuticos producidos a partir de estos, entre ellos, los denominados medicamentos monopolio del Estado y de control especial, y por tanto, requieren de una prescripción médica para su dispensación y administración. Asociado al uso irracional, el abuso de los medicamentos, principalmente de MF, se define como la "utilización excesiva, persistente o esporádica, de un medicamento de forma incongruente o desvinculada con la práctica médica admisible"14, es decir, el uso de medicamentos con una finalidad distinta a la de su indicación. En este sentido, algunos MF han sido incluidos en las Listas de Medicamentos de Alto Riesgo, considerados como los que presentan una mayor probabilidad de causar daños graves o incluso mortales a los pacientes, especialmente cuando se utilizan incorrectamente, entre ellos, opioides, benzodiacepinas, antipsicóticos, anestésicos y antiepilépticos ${ }^{15,16}$.

En Colombia no se dispone de información de estudios relacionados con la situación actual de la utilización de MF en el ámbito ambulatorio, que abarque, entre otros aspectos, los procesos y actores de la cadena del medicamento. Por lo anterior, es necesario conocer la situación actual de los MF en el contexto del sistema de salud colombiano, y de esta forma, tener un referente para la generación de nuevos estudios e intervenciones enfocadas en esta problemática. En este sentido, los objetivos de este estudio fueron: 1) caracterizar los establecimientos farmacéuticos; 2) describir los procesos técnicos, administrativos y asistenciales que se relacionan con la utilización de MF a nivel ambulatorio, incluyendo el cumplimiento de la normatividad vigente, del EF y del PF; y 3) determinar el nivel de percepción del riesgo y las necesidades de educación continua del PF en la utilización de los MF.

\section{Metodología}

\section{Tipo de estudio}

Estudio observacional descriptivo de corte transversal, durante el periodo mayo a septiembre de 2016.

\section{Población de estudio}

La población encuestada estuvo compuesta por el personal farmacéutico que labora en los Establecimientos Farmacéuticos (EF) ambulatorios (Droguerías y Farmacias-Droguerías) de Medellín y el Área Metropolitana (Colombia), entre ellos, Químicos Farmacéuticos (QF), Tecnólogos en Regencia de Farmacia (TRF), Técnicos profesionales en Servicios Farmacéuticos, Auxiliares de Servicios Farmacéuticos, Auxiliares de Farmacia y Expendedores de Drogas (denominación dada por el Ministerio de Salud a aquellas personas que no tienen formación universitaria, bien sea técnica o profesional, y con 25 de 
años edad como mínimo, experiencia en farmacias y/o droguerías de mínimo 10 años, y no requieren cursos de capacitación previos. También son denominados Director de Droguerías) ${ }^{17}$.

\section{Muestra}

Para la selección de la muestra se utilizó como marco muestral el listado de EF ambulatorios registrados en el ente regulador de vigilancia y control, que para el año 2015 registraba 1.389 EF. Se calculó un tamaño de muestra a conveniencia (no probabilístico) y los EF se seleccionaron aleatoriamente.

\section{Criterios de selección}

Se tuvieron en cuenta los EF de Medellín y Área Metropolitana. Se entrevistó al PF que se encontrará laborando en el EF, al momento de la visita y que desempeñará funciones propias del $\mathrm{PF}$ en el establecimiento.

\section{Instrumento y técnica utilizados para recolección de la información}

Se construyó un cuestionario que indagó por: 1) información del EF: municipio/barrio, nombre del EF/razón social, estrato socioeconómico, año de apertura, años de funcionamiento, horario de atención al público, número de empleados que laboran en el EF, tipo de EF (Droguería o Farmacia-Droguería) y servicios asistenciales que se ofrecen; 2) Información del PF: perfil técnico o profesional del propietario y del responsable o Director Técnico ante el ente regulador de vigilancia y control, institución de educación superior que es egresado y año de graduación; 3) información sobre los MF: MF que comercializa y su frecuencia de dispensación, y forma de adquisición de los productos farmacéuticos; 4) información sobre la percepción y formación continua del PF: percepción del PF idóneo para la dispensación de MF, educación continua, necesidades de capacitación, percepción del riesgo sobre la utilización de medicamentos fiscalizados, y percepción en el manejo que se le da a los MF en la cadena del medicamento.

Para obtener la información sobre los MF que los PF dispensan, específicamente los de mayor dispensación con o sin fórmula médica, se les presentó un listado actualizado de los MF aprobados por el ente regulador para su comercialización a nivel ambulatorio, este listado fue construido por los investigadores. Para evaluar la percepción del riesgo que el PF tiene sobre la utilización de los MF, se realizó el siguiente proceso: 1) se les presentó un listado de 5 medicamentos (morfina, tramadol, zolpidem, lovastatina y metoprolol), y de estos, 3 de ellos eran MF, 1 medicamento antihipertensivo y 1 medicamento hipolipemiante, seguido a esto; y 2) se les invitó a señalar los 3 medicamentos que ellos consideraban que su utilización podría generar mayor riesgo. Se incluyeron estos medicamentos, debido a que tienen una alta rotación y alta frecuencia de uso en el contexto del sistema de salud colombiano, principalmente, en el ámbito ambulatorio y por tratarse enfermedades crónicas, con ello, se pretendió establecer si el PF diferenciaba el riesgo entre MF y otros medicamentos de alto consumo/rotación utilizados en enfermedades crónicas.

Se realizaron encuestas personalizadas para la recolección de la información que fueron realizadas por cinco personas con experiencia en mercadeo y ventas, a quienes se capacitó en una sesión de entrenamiento teórico y práctico de cuatro horas. Adicionalmente, se realizó una prueba piloto en una muestra significativa de la misma población, con la que se puso a prueba el cuestionario, la encuesta, y el encuestador; y los investigadores dieron apoyo técnico durante todo el tiempo que duró la recolección de la información. Uno de los investigadores, actuó como coordinador de los encuestadores, quien además de establecer las rutas y las cargas de trabajo a cada encuestador, realizó contactos permanentes con los encuestadores para supervisar el trabajo. Finalmente, de forma periódica, se realizó muestreos aleatorios de los cuestionarios tabulados en búsqueda de errores o incongruencias.

\section{Análisis estadístico}

El análisis estadístico se realizó utilizando el programa SPSS 22 (SPSS-IBM INC, Chicago, IL, USA). Las variables cualitativas se presentan como porcentaje y las cuantitativas como media con su desviación estándar e intervalo de confianza del 95\%. El análisis bivariado se realizó utilizando la prueba Chi cuadrado, y la significancia estadística se estableció en $\mathrm{p}<0,05$. La percepción en el manejo que se le da a los MF en diferentes puntos de la cadena del medicamento se evaluó con una escala tipo Likert de 1 a 5 (siendo 1 muy deficiente, 2 deficiente, 3 regular, 4 bueno, y 5 excelente). Algunas de las variables se categorizaron para su análisis, como el año de apertura del EF (0 a 5 años, 6 a 10 años, 11 a 15 años, 16 a 20 años, 21 a 25 años, 26 a 30 años, y más de 30 años) y horas al día de atención al público (8 a 10 horas, 11 a 12 horas, 13 a 14 
horas, y más de 15 horas), el estrato socioeconómico del EF (estratos 1-2 y otros estratos), el perfil profesional del responsable (con título tecnológico y profesional, y sin tu título), y la percepción en el manejo que se le da a los MF en diferentes puntos de la cadena (1-3 percepción inadecuada, y 4-5 percepción adecuada).

\section{Consideraciones éticas}

El estudio fue avalado por el Comité de Ética de la Investigación de la Facultad Nacional de Salud Pública de la Universidad de Antioquia (Colombia). La participación del PF fue voluntaria y se obtuvo el respectivo consentimiento informado.

\section{Resultados}

Se visitaron $700 \mathrm{EF}$ durante los meses de mayo a septiembre de 2016 y se entrevistó igual cantidad de $\mathrm{PF}$, equivalente a una persona por $\mathrm{EF}$.

\section{Información sobre los Establecimientos Farmacéuticos}

De los resultados relacionados con la información sobre el EF, se destaca que el $88 \%$ (617) de los EF estaban ubicados en estratos 1, 2 y 3 (bajo y medio), y llevan en promedio 15,3 años de funcionamiento (SD 13,6 años). En promedio laboran tres personas por EF, y están abiertos para la atención al público 13 horas al día (SD 1,4 horas). Adicionalmente, el 92,6\% (648) de los EF comercializan dispositivos médicos; el $83,4 \%$ (584) ofrecen el servicio de inyectología; y el $34,7 \%$ (243) ofrece el servicio de domicilios (distribución de los medicamentos a los hogares). Se indagó por otros servicios, como la toma de presión arterial, la dispensación de medicamentos en convenio con prestadores o aseguradoras de salud, y el ofrecimiento de consulta médica, encontrándose que menos del 1\% de los EF ofrece alguno de estos servicios adicionales.

\section{Información sobre el Personal Farmacéutico}

En la Tabla 1 se describen los perfiles técnico o profesional del propietario y del responsable (Director Técnico) del EF. El 55,7\% (390) de los responsables o Directores Técnicos del EF ante el ente regulador de vigilancia y control eran Expendedores de Droguerías. El 48,8\% (448) de los propietarios laboran en el EF. Se encontró que el PF tiene en promedio 14 años de experiencia (SD 11,3 años).
Tabla 1. Perfil profesional del propietario y del responsable (Director Técnico) del Establecimiento Farmacéutico $(\mathrm{N}=700)$.

\begin{tabular}{|c|c|c|}
\hline & $\begin{array}{l}\text { Frecuencia } \\
\text { (n) }\end{array}$ & $\begin{array}{c}\text { Porcentaje } \\
(\%)\end{array}$ \\
\hline \multicolumn{3}{|l|}{ Perfil profesional del propietario } \\
\hline Expendedor de Droguería & 264 & 37,7 \\
\hline $\begin{array}{l}\text { Tecnólogos en Regencia de } \\
\text { Farmacia }\end{array}$ & 149 & 21,3 \\
\hline $\begin{array}{l}\text { Sin título universitario o } \\
\text { tecnológico }\end{array}$ & 98 & 14 \\
\hline $\begin{array}{l}\text { Administradores, contadores y } \\
\text { economistas }\end{array}$ & 50 & 7,1 \\
\hline $\begin{array}{l}\text { Otras profesiones diferentes al área } \\
\text { de la salud }\end{array}$ & 45 & 6,4 \\
\hline Médicos & 17 & 2,4 \\
\hline Químicos Farmacéuticos & 9 & 1,3 \\
\hline $\begin{array}{l}\text { Técnico Profesional en Servicio } \\
\text { Farmacéutico }\end{array}$ & 9 & 1,3 \\
\hline $\begin{array}{l}\text { Ingenierías (Sistemas, productividad } \\
\text { y calidad) }\end{array}$ & 4 & 0,6 \\
\hline Otros profesionales de la salud & 3 & 0,4 \\
\hline No reportado & 52 & 7,4 \\
\hline \multicolumn{3}{|c|}{ Perfil profesional del responsable (Director Técnico) del EF } \\
\hline Expendedor de droguería. & 390 & 55,7 \\
\hline $\begin{array}{l}\text { Tecnólogos en Regencia de } \\
\text { Farmacia }\end{array}$ & 231 & 33 \\
\hline Químicos Farmacéuticos & 9 & 1,3 \\
\hline $\begin{array}{l}\text { Técnico profesional servicio } \\
\text { farmacéutico }\end{array}$ & 7 & 1 \\
\hline $\begin{array}{l}\text { Sin título universitario o } \\
\text { tecnológico }\end{array}$ & 6 & 0,9 \\
\hline Otras profesiones & 4 & 0,6 \\
\hline Médicos & 1 & 0,1 \\
\hline No reportado & 52 & 7,4 \\
\hline
\end{tabular}

\section{Información sobre los Medicamentos} Fiscalizados

En relación a los MF, se encontró que el promedio de productos farmacéuticos vendidos al día fue 235 (SD 377), mientras que el de dispensaciones al día con y sin formula médica fue de 103 (SD 106), de las cuales el $82 \%$ (85) fueron dispensadas sin prescripción médica; esta información fue descrita directamente por el PF, y no fue posible corroborarla con las fórmulas médicas o de los registros de dispensación o facturación. Los MF que los PF describen que más se dispensan, fueron en su orden: amitriptilina 91,4\% (640), tramadol $90 \%$ (630), trazodona 60\% (419), ketotifeno $51,1 \%$ (351), imipramina $16,6 \%$ (116), dihidrocodeina $12,1 \%$ (85) y codeína $8,1 \%$ (57). Por su parte, el 
91,9\% (643) de los EF adquieren los medicamentos a distribuidores mayoristas, el 16,4\% (115) por intermedio de cooperativas de medicamentos, el 2,3\% (16) a distribuidores minoristas, y el 1,1\% (8) lo hace directamente a los laboratorios.

\section{Información sobre la percepción y la formación continuada del Personal Farmacéutico}

Con relación a la percepción de algunos aspectos claves en la utilización adecuada de MF por el PF y de la información que se brinda a los pacientes en la dispensación, se encontró que el 76,6\% (536) de los encuestados consideró que el Tecnólogo en Regencia de Farmacia es el personal más idóneo para dispensar MF, seguido del Expendedor de Droguerías en un 71,6\% (501), y en tercer lugar por el Químico Farmacéutico en un 63\% (441) (Tabla 2). Alrededor del 25\% (177) de los encuestados percibió como muy deficiente a regular el proceso enfocado en la información sobre el uso adecuado de los MF y la educación para la salud que se brinda a los usuarios y pacientes, y el 32\% (223) tuvo una percepción similar en la responsabilidad que tienen con el uso de los medicamentos y los resultados en salud. Adicionalmente, más de la tercera parte de los encuestados, $36 \%$ de ellos (257), consideró de muy deficiente a regular el proceso enfocado en la selección de los MF. En la Tabla 3 se presentan los hallazgos sobre la percepción de otros aspectos claves en la cadena del medicamento.

Tabla 2. Percepción del personal farmacéutico sobre el perfil e idoneida para dispensar medicamentos fiscalizados $(\mathrm{N}=700)$

\begin{tabular}{lcccc}
\hline & $\begin{array}{c}\text { Frecuencia } \\
\text { (n) }\end{array}$ & \multicolumn{2}{c}{$\begin{array}{c}\text { Porcentaje } \\
(\%)\end{array}$} \\
\hline Tecnólogos regencia de farmacia & 536 & 164 & 76,6 & 23,4 \\
Expendedor de droguería & 501 & 199 & 71,6 & 28,4 \\
Químicos farmacéuticos & 441 & 259 & 63,0 & 37,0 \\
Médico & 309 & 391 & 44,1 & 55,9 \\
Auxiliar de farmacia y droguería & 137 & 563 & 19,6 & 80,4 \\
Técnico de droguería & 103 & 597 & 14,7 & 85,3 \\
\hline
\end{tabular}

Tabla 3. Como califica el personal farmacéutico la utilización que se le da a los Medicamentos Fiscalizados, en los siguientes aspectos $(\mathrm{N}=700)$

\begin{tabular}{lcccccccccc}
\hline & Muy deficiente & \multicolumn{1}{c}{ Deficiente } & \multicolumn{2}{c}{ Regular } & \multicolumn{3}{c}{ Bueno } & \multicolumn{2}{c}{ Excelente } \\
\hline & $\boldsymbol{n}$ & $\boldsymbol{\%}$ & $\boldsymbol{n}$ & $\boldsymbol{\%}$ & $\boldsymbol{n}$ & $\boldsymbol{\%}$ & $\boldsymbol{n}$ & $\boldsymbol{\%}$ & $\boldsymbol{n}$ & $\%$ \\
\hline Selección & 149 & 21,3 & 23 & 3,3 & 85 & 12,1 & 65 & 9,3 & 378 & 54,0 \\
Adquisición & 14 & 2,0 & 14 & 2,0 & 74 & 10,6 & 63 & 9,0 & 530 & 75,7 \\
Recepción & 0 & 0 & 4 & 0,6 & 77 & 11,0 & 74 & 10,6 & 530 & 75,7 \\
Almacenamiento & 0 & 0 & 3 & 0,4 & 64 & 9,1 & 72 & 10,3 & 544 & 77,7 \\
Distribución & 4 & 0,6 & 14 & 2,0 & 73 & 10,4 & 72 & 10,3 & 521 & 74,4 \\
Dispensación & 3 & 0,4 & 3 & 0,4 & 72 & 10,3 & 81 & 11,6 & 525 & 75,0 \\
Información para su uso adecuado & 20 & 2,9 & 23 & 3,3 & 144 & 20,6 & 65 & 9,3 & 434 & 62,0 \\
Responsabilidad con el uso y resultados en salud & 59 & 8,4 & 62 & 8,9 & 109 & 15,6 & 46 & 6,6 & 411 & 58,7 \\
Manejo y rotación de inventarios & 0 & 0 & 2 & 0,3 & 63 & 9,0 & 68 & 9,7 & 550 & 78,6 \\
\hline
\end{tabular}

Se realizó un análisis ajustado (Tabla 4), utilizando como variables de respuesta: 1) la percepción del PF (adecuada o inadecuada), con relación a la selección de los MF como punto clave en la cadena del medicamento, 2) la información brindada sobre el uso adecuado de los MF y la educación para la salud a los usuarios y pacientes, y 3) la responsabilidad que tiene el PF con el uso de los medicamentos y los resultados en salud de los pacientes. Estas variables fueron valoradas como muy deficiente a regular en el análisis descriptivo. Se encontraron diferencias estadísticamente significativas en la percepción de la selección de los MF con el estrato socioeconómico del $\mathrm{EF}$ (valor $\mathrm{p}<0.001$ ). Adicionalmente, se encontró que la percepción en la información sobre el uso adecuado de los MF y la educación para la salud que brinda el PF a los usuarios y pacientes, varía de acuerdo con en el nivel profesional del PF responsable ante el ente regulador (valor $\mathrm{p}=0.037$ ), y la percepción en la responsabilidad que tiene el PF con el uso de los medicamentos y los resultados en salud de los pacientes (valor $\mathrm{p}=0,049$ ). 
Tabla 4. Relación de la percepción del personal farmacéutico de algunos aspectos claves en la utilización adecuada de Medicamentos Fiscalizados.

\begin{tabular}{|c|c|c|c|c|c|}
\hline Percepción & Valoración & $\begin{array}{l}\text { Estrato } \\
\text { socioeconómico } 1 \text { y } 2\end{array}$ & Valor $\mathbf{p}$ & $\begin{array}{c}\text { Nivel profesional del } \\
\text { responsable (TRF/QF) }\end{array}$ & Valor $\mathbf{p}$ \\
\hline \multirow{2}{*}{$\begin{array}{l}\text { Percepción del PF con relación a la } \\
\text { selección de los MF }\end{array}$} & Adecuada & 136 & \multirow{2}{*}{0.001} & 181 & \multirow{2}{*}{0.362} \\
\hline & Inadecuada & 42 & & 54 & \\
\hline \multirow{2}{*}{$\begin{array}{l}\text { Percepción del PF sobre la información } \\
\text { brindada para el uso adecuado de los MF }\end{array}$} & Adecuada & 143 & \multirow{2}{*}{0.119} & 153 & \multirow{2}{*}{0.037} \\
\hline & Inadecuada & 38 & & 87 & \\
\hline \multirow{2}{*}{$\begin{array}{l}\text { Percepción de la responsabilidad que tiene } \\
\text { el PF con el uso de los medicamentos y los } \\
\text { resultados en salud de los pacientes }\end{array}$} & Adecuada & 125 & \multirow{2}{*}{0.13} & 167 & \multirow{2}{*}{0.049} \\
\hline & Inadecuada & 53 & & 69 & \\
\hline
\end{tabular}

TRF: Tecnólogo en Regencia de Farmacia; QF: Químico Farmacéutico

Con relación a la educación continua, se encontró que solo el 20,1\% (141) del PF ha realizado algún tipo de estudio, capacitación y/o actualización luego de haberse graduado del pregrado. Relacionado con las necesidades de formación, del total de la muestra, el $48,4 \%$ (339) consideró necesario profundizar en temas relacionados con la administración y gestión de los SF; el 81,2\% (569) pensaba que deben actualizarse $\mathrm{y}$ adquirir competencias en actividades asistenciales relacionadas con la atención al paciente y usuario; $44,9 \%$ (314) de los encuestados manifestaron la necesidad de profundizar en temas inspección, vigilancia y control de medicamentos; solo el $41 \%$ (287) ven necesario mejorar sus competencias sobre los MF y su utilización, y se resalta que apenas el 4,4\% (31) del PF se ha capacitado o ha recibido algún tipo de educación continua en este grupo farmacológico de medicamentos, con un promedio de 40 horas totales de capacitación.

\section{Discusión}

Con esta caracterización se logró conocer la información básica y la distribución de los EF de Medellín y el Área Metropolitana, abarcando más del $50 \%$ (700/1.389) de los EF minoristas registrados en esta zona, compuesto por 10 municipios con alrededor de 3.777.009 habitantes (datos del 2015) ${ }^{18}$, aportando información sobre la distribución y el contexto socioeconómico de las áreas urbanas en la cuales los EF tienen inferencia dentro del sistema de salud, que en su gran mayoría se encuentran en los estratos bajo y medio. Solo se encontró un estudio similar que caracterizó los establecimientos farmacéuticos en Urabá (Colombia) ${ }^{19}$, aunque este no fue el objetivo principal del estudio. Los autores encontraron que, de los 147 establecimientos encuestados, 35 (24\%) son establecimientos comerciales, 31 (89\%) son almacenes de cadena y 4 (11\%) almacenes de grandes superficies. Por su parte, 92 (63\%) corresponden a servicios farmacéuticos independientes, y de estos 89 (97\%) son establecimientos minoristas y solo $3(3 \%)$ son mayoristas; 20 (14\%) son servicios farmacéuticos dependientes, 2 (10\%) son de alta complejidad, $6(30 \%)$ de mediana y $12(60 \%)$ de baja.

El panorama actual del ejercicio de la farmacia en Colombia y en especial de la utilización de MF, requiere un alto grado de formación, profesionalización y educación continua, preferiblemente basada en competencias (conocimientos, habilidades y actitudes), de acuerdo a las recomendaciones de la Organización Mundial de la Salud (OMS) y la Federación Internacional Farmacéutica (FIF) ${ }^{7,20}$; sin embargo, los hallazgos de este estudio demuestran que la mayoría de los EF ambulatorios se encuentran dirigidos por personal sin formación universitaria o técnica, en su gran mayoría es personal empírico autorizado para el ejercicio de la farmacia, además, de no contar con evidencias de capacitación y educación continua. Datos similares se describen en los resultados de la caracterización realizada en Urabá (Colombia) ${ }^{19}$, con relación al grado académico del propietario del establecimiento, tan solo 16 (11\%) eran egresados de TRF, 24 (16\%) eran Expendedores de Droguería y 33 (22\%) eran propietarios con un título diferente al área de la salud. Con relación a si el propietario laboraba en el establecimiento, 66 (45\%) de los PF laboraban en el $\mathrm{EF}$, similar a lo evidenciado en este estudio.

Otro estudio realizado en Bogotá en el 2005, describió sobre el perfil y prácticas de los expendedores de medicamentos en EF ambulatorios, reportando que cerca del $67 \%$ de ellos no contaban con formación técnica o profesional y que, en más del $55 \%$ de los casos, el expendio de medicamentos se realizó de 
una manera inadecuada ${ }^{21}$. Ambos estudios describen un panorama crítico que relacionan la baja o poca competencia del PF que labora en los EF, a las necesidades técnico-administrativas y asistenciales en los procesos de la cadena del medicamento, y a factores externos como el estrato socioeconómico, entre otros. A pesar de contar con un marco normativo propicio para el desarrollo de las competencias del talento humano en salud, existen necesidades en la oferta de formación de PF con perfil tecnológico y profesional y la calificación del mismo, además, de la necesidad de un plan o programa estructurado de educación continua, sin embargo, estas necesidades y oportunidades ya se han comenzado a identificar ${ }^{22}$.

La dispensación de medicamentos es entendida como el acto profesional de la entrega del medicamento correcto, al usuario indicado, en el momento oportuno, acompañado de la información para el correcto uso del mismo y el cumplimiento de los objetivos terapéuticos buscados ${ }^{3,23}$. Vale la pena destacar que algunas empresas en Colombia relacionadas con la gestión de medicamentos, han implementado metodologías de farmacovigilancia para la identificación de efectos adversos o de posibles interacciones de los medicamentos prescritos, dichos sistemas están diseñados para realizar intervenciones posteriores al proceso de dispensación y/o entrega de medicamentos, específicamente en programas especiales gestionados por operadores logísticos y prestadores de servicios de salud, y financiados por las aseguradoras ${ }^{24}$, desafortunadamente este tipo de servicios y programas aún no se observan desde los EF ambulatorios, notándose falencias en la vigilancia y control de la correcta dispensación de medicamentos que requieren prescripción, como se notó en esta caracterización, de acuerdo a los PF encuestados el $82 \%$ de los MF son dispensados sin prescripción médica, proporción similar a lo reportado en otro estudio en el contexto colombiano que describe que la venta de antibióticos sin receta alcanzaría el $80 \%{ }^{25}$.

En la actualidad se han comenzado a plantear muchos desafíos y oportunidades para el PF a nivel mundial, y en particular aquellos que laboran a nivel ambulatorio. Aunque sus actividades principales son más administrativas, la dispensación de medicamentos cuando mucho se limita a la simple entrega o despacho de los medicamentos, sin embargo, desde hace algunos años la OMS y la FIF han impulsado su evolución hacia una farmacia más asistencial orientada al paciente ${ }^{7}$, jugando un rol crucial en la optimización de la farmacoterapia y mejorando los resultados de los pacientes, evitando al mismo tiempo el uso irracional de los medicamentos. Por tanto, con los hallazgos de este estudio se evidencia que los PF perciben que ellos tienen falencias en sus competencias para realizar de forma efectiva y oportuna los procesos enfocados a la información sobre el uso adecuado de los MF y la educación para la salud que se brinda a los usuarios y pacientes, y similarmente, esta percepción es la misma con la responsabilidad que tienen para alcanzar los objetivos y resultados en salud. Por ello, se puede justificar la necesidad de generar estrategias en pro de aumentar la cantidad de farmacéuticos (profesionales y tecnólogos) a nivel ambulatorio, además, de fortalecer sus competencias laborales para la utilización adecuada de medicamentos de grupos terapéuticos complejos, como lo son los MF, en parte, por el su uso irracional, y el riesgo de su abuso y dependencia.

De acuerdo con los resultados de esta caracterización, se pudo determinar que los MF de mayor dispensación a nivel ambulatorio son: antidepresivos, opioides y benzodiacepinas. Según datos del Instituto Nacional de Vigilancia de Medicamentos y Alimentos (INVIMA), de las 342 sustancias sometidas a fiscalización nacional e internacional, 257 (75\%) son materias primas y medicamentos fabricados a partir de estas pero que no tienen registro sanitario para su comercialización en Colombia. Para el 2015, 85 (25\%) de las sustancias de este listado eran MF que contaban con registro sanitario, de ellas, 29 (9\%) tenían el registro vencido, y 56 (16\%) tenían el registro vigente para su comercialización en el país. Para esa fecha, se notaba un crecimiento en la cantidad de registros sanitarios aprobados de algunos $\mathrm{MF}$, por ejemplo, para el tramadol existían 85 registros diferentes.

El PF que labora en EF de estratos bajos (estratos 1 y 2) consideran que tienen falencias técnicas y administrativas enfocadas en uno de los procesos más críticos de la cadena del medicamento, como lo es la selección de los medicamentos, que realizado de forma adecuada, contribuye a disminuir la ocurrencia de errores en los procesos subsecuentes de la cadena, y de acuerdo a la $\mathrm{PFN}^{2}$, en el contexto del sistema de salud colombiano, existen debilidades en la selección de medicamentos y en la definición del plan de beneficios, y sumado a la insuficiente formación y capacitación continua del $\mathrm{PF}$, a la poca información técnica de consulta pública, debilita la coherencia entre la normatividad y el uso adecuado de medicamentos. Además, el PF con título universitario de TRF o 
QF tiene una mayor percepción de la importancia de brindar información adecuada y oportuna a sus pacientes, y su responsabilidad como profesionales de la salud en la utilización adecuada de los medicamentos y los resultados en salud.

Los resultados de este estudio, específicamente en lo relacionado con el riesgo de los MF por parte del PF, describe que ellos saben, entienden y perciben que juegan un papel importante en la promoción del uso adecuado de los MF y la prevención del abuso de estos. Sin embargo, la alta frecuencia de dispensaciones de medicamentos sin formula médica indica que, aunque se percibe el riesgo esto podría no ser suficiente para mejorar su utilización.

\section{Limitaciones}

Una de las limitaciones fue la realización de un muestreo no probabilístico. Además, la recolección de la información fue realizada por personal cuyo perfil profesional era de ventas y mercadeo, sin embargo, se minimizó esta limitación con la capacitación por parte de los investigadores a los encuestadores y se brindó apoyo técnico durante toda la recolección de la información, igualmente los instrumentos no fueron validados, con lo que se reconocen las limitaciones en la validez interna y externa de los resultados.

\section{Conclusiones}

Se identifica la información general de los EF, al igual que de los procesos relacionados con la utilización de MF, como son los procesos técnicos, administrativos y asistenciales enfocados en la utilización de estos medicamentos. Es de recalcar, que este estudio se realizó a más del $50 \%$ de los EF minoristas de Medellín y el Área Metropolitana, describiendo los perfiles técnicos y profesionales de los propietarios y los responsables de los EF ante el ente regulador. Por otro lado, la percepción de los encuestados sobre el PF idóneo para la dispensación correcta de MF podría ser inadecuada. Un alto porcentaje del PF percibe falencias de educación enfocada en la utilización correcta de los MF. En la revisión bibliográfica realizada no se encontraron estudios enfocados en caracterizar los EF ambulatorios, y en especial en determinar las necesidades y percepciones del PF con relación a las competencias laborales y el riesgo en la utilización de MF, es por ello, que estos resultados pueden servir para generar futuros estudios que se enfoquen en la problemática del uso racional y el abuso de los MF a nivel ambulatorio, o con objetivos similares.

\section{Agradecimientos}

A Laboratorios Laproff S.A por el apoyo logístico y técnico para la realización de las encuestas, a la Facultad de Ciencias Farmacéuticas y Alimentarias de la Universidad de Antioquia.

\section{Consideraciones éticas}

El estudio fue avalado por el Comité de Ética de la Investigación de la Facultad Nacional de Salud Pública de la Universidad de Antioquia (Colombia). La participación del PF fue voluntaria y se obtuvo el respectivo consentimiento informado.

\section{Financiación}

El grupo Promoción y Prevención Farmacéutica recibió financiación de la convocatoria de sostenibilidad 20142015 del Comité para el Desarrollo de la Investigación de la Universidad de Antioquia.

\section{Conflicto de interés}

Los autores declaran no tener ningún conflicto de intereses en el trabajo realizado.

\section{Referencias}

1. República de Colombia. Ministerio de la Protección Social-Organización Panamericana de la Salud. Política Farmacéutica Nacional. 2003.

2. Consejo Nacional de Política Económica y Social, Departamento Nacional de Planeación, Republica de Colombia. Politica Farmaceutica Nacional Documento Compes Social 155. Conpes Soc Cons Nac política económica y Soc. 2012;33.

3. República de Colombia. Ministerio de Protección Social. Decreto 2200 de 2005. Reglamentación del servicio farmacéutico y se dictan otras disposiciones. 2005. p. 1-15.

4. Colegio Nacional de Químicos Farmacéuticos - Servicio Nacional de Aprendizaje, SENA. Caracterización ocupacional del sector farmacéutico en Colombia, enfoque por entornos. Servicio Nacional de Aprendizaje - SENA; 2008.

5. International Medical Statistics, IMS.

6. Amau LJ. Principios de Epidemiología del Medicamento. Cap. 3. Promoción del Uso Racional de Medicamentos y Preparación de Guías Clínicas. Segunda ed. Barcelona; 1993.

7. Organización Mundial de la Salud, OMS. Declaración de Tokio. El papel del farmacéutico 
en el sistema de atención de salud. Informe de La Reunión de la OMS Tokio, Japón, 31 de agosto al 3 de septiembre de 1993. Buenas prácticas de farmacia: normas de calidad de servicios farmacéuticos.

8. Organización Mundial de la Salud, Federación Farmacéutica Internacional. OMS/FIP. Directrices Conjuntas FIP/OMS sobre Buenas Prácticas en Farmacia: Estándares para la calidad de los servicios farmacéuticos. 2011.

9. Otero MJ, Martín R, Santos B, Puigventós F. Farmacia hospitalaria: órgano oficial de expresión científica de la Sociedad Española de Farmacia Hospitalaria. Farmacia Hospitalaria. Ed. Garsi; 264270 p.

10. About Medication Errors | NCC MERP.

11. Farcas A, Sinpetrean A, Mogosan C, Palage M, Vostinaru O, Bojita $\mathrm{M}$, et al. Adverse drug reactions detected by stimulated spontaneous reporting in an internal medicine department in Romania. Eur J Intern Med. 2010; 21(5): 453-457. DOI: 10.1016/j. ejim.2010.05.014.

12. Wu T-Y, Jen M-H, Bottle A, Molokhia M, Aylin P, Bell D, et al. Ten-year trends in hospital admissions for adverse drug reactions in England 1999-2009. J R Soc Med. 2010; 103(6): 239-250. DOI: 10.1258/ jrsm.2010.100113.

13. Organización Mundial de la Salud, OMS. Garantizando el equilibrio en las políticas nacionales sobre sustancias fiscalizadas. 2012.

14. República de Colombia. Ministerio de ambiente vivienida y desarrollo terrritorial. Resolución 1478. 2006.

15. Organización Mundial de la Salud, OMS. Informe 34. Comité de expertos en farmacodependencia. 2004.

16. Office of applied studies substances abuse and menthal health services administration. National survey on drug use and health. 2013.

17. ISMP España. http://www.ismp-espana.org/ documentos/view/39.

18. República de Colombia. Ministerio de salud. Decreto 1070. 1990.

19. Área Metropolitana. http://www.metropol.gov.co/ institucional/Paginas/IndicadoresGestion.aspx.

20. Arroyave O, Álvarez W, Amariles P, Vásquez M, Cardona B. Pertinencia laboral y social del programa de Tecnología en Regencia de Farmacia en la región de Urabá. Rev Univ Ind Santander Salud. 2016; 48(4): 526-534. DOI: http://dx.doi.org/10.18273/ revsal.v48n4-2016011.

21. Pharmacy education taskforce a global competency framework. Federation International Pharmaceutical FIP. 2012.
22. Vacca C, Orozco J, Figueras A, Capellà D. Assessment of risks related to medicine dispensing by nonprofessionals in Colombia: clinical case simulations. Ann Pharmacother. 2005; 39(3): 527532.

23. Ceballos JM, Amariles P. Needs and opportunities for outpatient pharmacies in colombia: the case of continuing education programs for pharmacists. Rev Vitae. 2017; 24(1): 9-11.

24. Consejo General de Colegios, Ofi iales de Farmacéuticos. Grupo de Trabajo de Buenas Prácticas. Servicio de Dispensación de medicamentos y productos sanitarios. Buenas Prácticas en Farmacia Comunitaria en España. 2014.

25. Ifarma. Desarrollo de los servicios farmacéuticos en Colombia: Las empresas de gestión de servicios farmacéuticos. 2013.

26. Vacca CP, Niño CY, Reveiz L. Restriction of antibiotic sales in pharmacies in Bogotá, Colombia: a descriptive study. Rev Panam Salud Pública. 2011; 30(6): 586-591. 